\title{
The Bernstein Form of a Polynomial
}

\author{
G. T. Cargo* \\ Syracuse University, Syracuse, New York \\ and \\ O. Shisha** \\ Aerospace Research Laboratories, Wright-Patterson AFB, Ohio
}

(October 27, 1965)

\section{Introduction}

Let $P$ denote a polynomial with real coefficients. In this paper we devise an algorithm for determining upper and lower bounds for the set $\{P(x): 0 \leqslant x \leqslant 1\}$.

\section{Some Preliminary Observations}

For a polynomial $P$ with real coefficients, it is possible, given $\epsilon>0$, to compute, by means of arithmetical operations whose number can be readily determined, upper and lower bounds for $\{P(x): 0 \leqslant x \leqslant 1\}$ which differ from the corresponding sharp bounds by less than $\epsilon$. This is an immediate consequence of the simple observation that, if $\mathrm{P}(\mathrm{x}) \equiv \mathrm{a}_{0}+\mathrm{a}_{1} \mathrm{x}$ $+\ldots+\mathrm{a}_{\mathrm{n}} \mathrm{x}^{\mathrm{n}}(\mathrm{n} \geqslant 1)$ is a polynomial with real coefficients, then, for each positive integer $\mathrm{m}$,

$$
\begin{aligned}
\min \{\mathrm{P}(\mathrm{k} / \mathrm{m}): \mathrm{k}=1,2, \ldots, \mathrm{m}\} & -\frac{1}{\mathrm{~m}} \sum_{k=1}^{n} \mathrm{k}\left|\mathrm{a}_{\mathrm{k}}\right| \\
\leqslant \mathrm{P}(\mathrm{x}) \leqslant \max \{\mathrm{P}(\mathrm{k} / \mathrm{m}): \mathrm{k} & =1,2, \ldots, \mathrm{m}\} \\
& +\frac{1}{\mathrm{~m}} \sum_{\mathrm{k}=1}^{\mathrm{n}} \mathrm{k}\left|\mathrm{a}_{\mathrm{k}}\right|(0 \leqslant \mathrm{x} \leqslant 1),
\end{aligned}
$$

which follows from the law of the mean and the inequality $\left|P^{\prime}(\mathrm{x})\right| \leqslant \sum_{k=1}^{n} \mathrm{k}\left|\mathrm{a}_{\mathrm{k}}\right|(0 \leqslant \mathrm{x} \leqslant 1)$.

This procedure has the disadvantage that an extremely large number of arithmetical operations may be required to obtain moderately good bounds. In this paper we consider an algorithm which sacrifices accuracy in order to gain tractability.

*The research of this author was supported by the National Science Foundation through grant GP 1086. Present address: Syracuse University, Syracuse, N.Y.

${ }^{* *}$ United States Air Force, Wright-Patterson Air Force Base, Ohio.

\section{The Bernstein Form of a Polynomial}

Theorem. Let $\mathrm{P}(\mathrm{x}) \equiv \mathrm{a}_{0}+\mathrm{a}_{1} \mathrm{x}+\ldots+\mathrm{a}_{\mathrm{n}} \mathrm{x}^{\mathrm{n}}$ be $a$ polynomial of exact degree $\mathrm{n}(\geqslant 0)$ with real coefficients. Then

$$
\begin{aligned}
& \min \left\{b_{\mathrm{k}}: \mathrm{k}=0,1, \ldots, \mathrm{n}\right\} \leqslant \mathrm{P}(\mathrm{x}) \\
& \quad \leqslant \max \left\{\mathrm{b}_{\mathrm{k}}: \mathrm{k}=0,1, \ldots, \mathrm{n}\right\} \quad(0 \leqslant \mathrm{x} \leqslant 1)
\end{aligned}
$$

where

$$
\mathrm{b}_{\mathrm{k}}=\sum_{r=0}^{\mathrm{k}} \mathrm{a}_{\mathrm{r}}\left(\begin{array}{l}
\mathrm{k} \\
\mathrm{r}
\end{array}\right) /\left(\begin{array}{l}
\mathrm{n} \\
\mathrm{r}
\end{array}\right) \quad(\mathrm{k}=0,1, \ldots, \mathrm{n}) .
$$

The upper (lower) bound is sharp if and only if it is equal to $\mathrm{b}_{0}$ or to $\mathrm{b}_{\mathrm{n}}$.

Preliminary remark. It is obvious from (2) that the bounds given by (1) are always at least as good as the crude bounds $\pm \sum_{k=0}^{\mathrm{n}}\left|a_{k}\right|$.

Proof: The following representation (to be established below, but essentially due to S. N. Bernstein ${ }^{1}$ ) holds:

$$
P(x) \equiv \sum_{k=0}^{n} \mathrm{~b}_{k}\left(\begin{array}{l}
n \\
k
\end{array}\right) x^{k}(1-x)^{n-k}
$$

where $b_{k}(k=0,1, \ldots, n)$ is given by $(2){ }^{2}$ Since $\sum_{k=0}^{n}\left(\begin{array}{l}n \\ k\end{array}\right) x^{k}(1-x)^{n-k} \equiv 1$ and $\left(\begin{array}{l}n \\ k\end{array}\right) x^{k}(1-x)^{n-k} \geqslant 0(0 \leqslant x$

\footnotetext{
${ }^{1}$ Bernstein, S. N., On the best approximation of continuous functions by polynomials of a given degree (Russian), Communications of the Khar'kov Mathematical Society, Series $2,13,49-194(1912)$

2 One easily sees that $\left(x^{k}(1-x)^{n-k}\right)_{k=0}^{n}$ forms a basis for the space of all polynomials $\sum^{n \cdot}$ $\sum_{k=0} c_{k} x^{k}, c_{k}$ real. Consequently, a representation (3) with real $b_{k}$ 's uniquely determined must hold.
} 
$\leqslant 1 ; k=0,1, \ldots, n),(3)$ implies (1). We refer to $P(x)$ written in the form $\sum_{k=0}^{n} b_{k}\left(\begin{array}{l}n \\ k\end{array}\right) x^{k}(1-x)^{n-k}$ as the "Bernstein form of $P(x)$." The last sum is the Bernstein polynomial of order $n$ of every real function $f$ defined on $[0,1]$ for which $f(k / n)=b_{k}(k=0,1, \ldots, n){ }^{3}$

If $\max \left\{b_{k}: k=0,1, \ldots, n\right\}$ is equal to $b_{0}$ or to $b_{n}$, then the upper bound given by (1) is sharp since $P(0)=b_{0}$ and $P(1)=b_{n}$. Conversely, if the upper bound given by (1) is sharp, then it is equal to $b_{0}$ or to $b_{n}$ since, if $0<x<1$, either $b_{0}=b_{1}=\ldots=b_{n}$ or $\sum_{k=0}^{n} b_{k}\left(\begin{array}{l}n \\ k\end{array}\right) x^{k}(1-x)^{n-k}<\max \left\{b_{k}: k=0,1, \ldots, n\right\}$.

The sharpness of the lower bound can be treated in an analogous fashion.

In order to establish (3), we observe that

$$
\begin{aligned}
\sum_{r=0}^{n} a_{r} x^{r} & \equiv \sum_{r=0}^{n} a_{r} x^{r}\{x+(1-x)\}^{n-r} \\
& \equiv \sum_{r=0}^{n} \sum_{s=0}^{n-r} a_{r}\left(\begin{array}{c}
n-r \\
s
\end{array}\right) x^{n-s}(1-x)^{s} \\
& \equiv \sum_{s=0}^{n} \sum_{r=0}^{n-s} a_{r}\left(\begin{array}{c}
n-r \\
s
\end{array}\right) x^{n-s}(1-x)^{s} \\
& \equiv \sum_{k=0}^{n}\left\{\sum_{r=0}^{k} a_{r}\left(\begin{array}{c}
n-r \\
n-k
\end{array}\right) /\left(\begin{array}{l}
n \\
k
\end{array}\right)\right\}\left(\begin{array}{l}
n \\
k
\end{array}\right) x^{k}(1-x)^{n-k} \\
& \equiv \sum_{k=0}^{n}\left\{\sum_{r=0}^{k} a_{r}\left(\begin{array}{l}
k \\
r
\end{array}\right) /\left(\begin{array}{l}
n \\
r
\end{array}\right)\right\}\left(\begin{array}{l}
n \\
k
\end{array}\right) x^{k}(1-x)^{n-k} .
\end{aligned}
$$

This completes the proof of the theorem.

Next, we seek a more efficient method than the direct use of formula (2) for computing $b_{k}(k=0$, $1, \ldots, n$ ). To this end, we note (footnote 3, p. 12) that, if $P(x) \equiv \sum_{r=0}^{n} b_{r}\left(\begin{array}{l}n \\ r\end{array}\right) x^{r}(1-x)^{n-r}(n \geqslant 1)$, then, as a simple calculation shows,

$$
P^{\prime}(x) \equiv n \sum_{r=0}^{n-1}\left\{\Delta b_{r}\right\}\left(\begin{array}{c}
n-1 \\
r
\end{array}\right) x^{r}(1-x)^{n-1-r} .
$$

Repeating this process, we obtain

$$
\begin{aligned}
P^{(k)}(x) \equiv & n(n-1) \ldots(n-k+1) \sum_{r=0}^{n-k}\left\{\Delta^{k} b_{r}\right\} \\
& \left(\begin{array}{c}
n-k \\
r
\end{array}\right) x^{r}(1-x)^{n-k-r}(k=1,2, \ldots, n) .
\end{aligned}
$$

Since $a_{k}=P^{(k)}(0) / k !(k=1,2, \ldots, n)$ if $P(x) \equiv a_{0}$

\footnotetext{
${ }^{3}$ Lorentz, G. G., Bernstein Polynomials, University of Toronto Press, Toronto, 1953.
}

$+a_{1} x+\ldots+a_{n} x^{n},(4)$ yields

$$
a_{k}=\left(\begin{array}{l}
n \\
k
\end{array}\right) \Delta^{k} b_{0} \quad(k=1,2, \ldots, n) .
$$

Equation (5) suggests a synthetic method for determining the Bernstein form of a polynomial $P(x)$ $\equiv a_{0}+a_{1} x+\ldots+a_{n} x^{n}\left(a_{r}\right.$ real, $\left.n \geqslant 1, a_{n} \neq 0\right)$ without using (2) explicitly. One simply writes the numbers $\Delta^{k} b_{0}=a_{k} /\left(\begin{array}{l}n \\ k\end{array}\right)(k=0,1, \ldots, n)$ in their usual positions in a difference table (see table 1) and then completes the table to obtain $b_{0}, b_{1}, \ldots ., b_{n}$. In view of (4), the completed difference table also yields bounds for $\left\{P^{(k)}(x): 0 \leqslant x \leqslant 1\right\}(k=1,2, \ldots ., n)$.

For example, if $P(x) \equiv 1+10 x-20 x^{2}+30 x^{3}+15 x^{4}$ $-x^{5}$, we start with the incomplete difference table (table 2) and complete it to obtain table 3 , which yields $b_{0}=1, \quad b_{1}=3, b_{2}=3, b_{3}=4, b_{4}=12$, and $b_{5}=35$. Table 3 also yields the bounds 0 and 115 for $P^{\prime},-40$ and 300 for $P^{\prime \prime}, 180$ and 480 for $P^{\prime \prime \prime}$, and 240 and 360 for $P^{(4)}$ on $[0,1]$.

TABLE 1

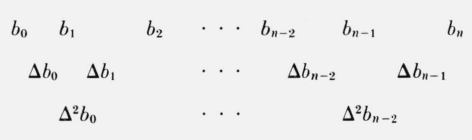

$\Delta^{n} b_{0}$

TABLE 2

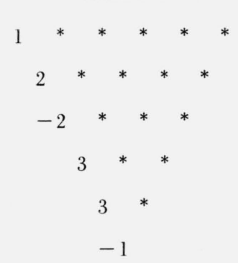

TABLE 3

$\begin{array}{llllll}1 & 3 & 3 & 4 & 12 & 35\end{array}$

$\begin{array}{lllll}2 & 0 & 1 & 8 & 23\end{array}$

$\begin{array}{llll}-2 & 1 & 7 & 15\end{array}$

$\begin{array}{lll}3 & 6 & 8\end{array}$

$3 \quad 2$

$-1$

\section{Conclusion}

We conclude with some miscellaneous remarks.

Formula (2) follows from (5) and the Newton interpolation formula.

One can obviously consider the "Bernstein form" $\sum_{k=0}^{n} b_{k}\left(\begin{array}{l}n \\ k\end{array}\right) x^{k}(1-x)^{n-k}$ of a polynomial $P$ with complex 
coefficients. It is clear that $P$ maps $[0,1]$ into the convex hull of the set $\left\{b_{0}, b_{1}, \ldots, b_{n}\right\}$.

In view of (5), it is easy to state simple sufficient conditions for a polynomial $P(x) \equiv a_{0}+a_{1} x+\ldots$. $+a_{n} x^{n} \equiv \sum_{r=0}^{n} b_{r}\left(\begin{array}{c}n \\ r\end{array}\right) x^{r}(1-x)^{n-r}$ with real coefficients to have certain properties. For example, if $n \geqslant 1$, $a_{0}>0$, and $k a_{k}+(n-k+1) a_{k-1}>0(k=1,2, . . ., n)$, then $P(x)>0$ whenever $0 \leqslant x \leqslant 1$. Indeed, $k a_{k}$ $+(n-k+1) a_{k-1}>0$ is equivalent to $\Delta^{k} b_{0}+\Delta^{k-1} b_{0}$ $=a_{k} /\left(\begin{array}{l}n \\ k\end{array}\right)+a_{k-1} /\left(\begin{array}{c}n \\ k-1\end{array}\right)>0$, and, consequently, all the entries in table 1 are positive except possibly $\Delta^{k} b_{0}(k=1,2, \ldots ., n)$.

Let $n$ be a positive integer. An analog of (1) obviously holds if, for each $k(k=0, \ldots, n)$, one takes instead of $\left(\begin{array}{l}n \\ k\end{array}\right) x^{k}(1-x)^{n-k}$ a polynomial $q_{k}(x) \equiv \sum_{r=0}^{n} c_{r}^{(k)} x^{r}$ with real coefficients such that (a) $q_{k}(x) \geqslant 0$ if $0 \leqslant x \leqslant 1$, (b) $q_{0}, q_{1}, \ldots, q_{n}$ are linearly independent, and (c) $\sum_{k=0}^{n} q_{k}(x) \equiv 1$. It is easy to verify that there are many such sequences $\left(q_{k}(x)\right)_{k=0}^{n}$ of polynomials. For example, for each $\alpha, 0<\alpha \leqslant 1$, the polynomials $P_{k}(x) \equiv\left(\begin{array}{c}n \\ k\end{array}\right)(\alpha x)^{k}(1-\alpha x)^{n-k} \quad(k=0, \quad 1, \quad \ldots . n)$ form such a sequence. Moreover, if $q_{0}, q_{1}, \ldots ., q_{n}$ form such a sequence, then so do $Q_{0}, Q_{1}, \ldots, Q_{n}$ where $Q_{k}(x) \equiv \frac{1}{x} \int_{0}^{x} q_{k}(t) d t \quad(k=0,1, \ldots, n)$.

(Paper 70B1-168) 